\title{
THE EUROPEAN CODE OF CONDUCT ON CLOUD SERVICES AND DATA PORTABILITY: WHAT CHANGES IN THE CLOUD ECOSYSTEM
}

\author{
Maro Androutsopoulou ${ }^{1}$, John Violos ${ }^{2}$, Dimitrios Askounis ${ }^{1}$, Theodora Varvarigou ${ }^{2}$, \\ Panagiotis Kokkinakos ${ }^{1}$ and Christos Kontzinos ${ }^{1}$ \\ ${ }^{I}$ Decision Support Systems Laboratory, School of Electrical and Computer Engineering, \\ National Technical University of Athens, 9, Heroon Polytechniou Str, 15773, Zografou, Greece \\ ${ }^{2}$ Electrical and Computer Engineering, National Technical University of Athens, \\ 9, Heroon Polytechniou Str, 15773, Zografou, Greece
}

\begin{abstract}
Cloud computing, IaaS Cloud Services and Data Portability are topics of great interest and largely contemplated lately not only because of the focus the academia and the research community is showing, but also of the enterprises and the ECs continuously growing interest. Even though the adoption of cloud computing by European enterprises specially is steadily growing, it has not been as big as it would have been expected due to specific barriers faced by Cloud Service Customers (CSCs) such as lack of trust, uncertainty about the applicable law, unclear compliance to existing schemes and legislation, as well as the lack of interoperability among service providers, of transparency in security aspects and of control of the data.

The work presented deliberates what effect a Code of Conduct (Code) for Data Portability and Cloud Service Switching for IaaS Cloud services would have if included in the Certification Scheme for Cloud Computing already being prepared by the EC, and if the impact will in fact be the increase of CSCs condense regarding porting and switching between IaaS cloud services, at a low cost and with minimal disruption. The economics of IaaS Cloud Services will play a significant role on how cloud computing will grow, so a review of available economic models used to forecast the economic impact of cloud computing will be presented, along with a proposal of a model that could potentially be used to forecast the economic footprint of a Code for Data Portability and Cloud Service Switching.
\end{abstract}

\section{KEYWORDS}

Code of Conduct, Cloud Infrastructure Services Providers, Data Portability, Cloud Services Switching

\section{INTRODUCTION}

The Regulation of the European parliament and of the council of 14 November 2018 on a framework for the free flow of non-personal data in the European Union sets the principles for the cloud switching and the transfer of non-personal data between two different Cloud Infrastructure Services Providers (CISP), or between the cloud customer on-premises facilities and a CISP. The purpose of the Regulation is to: "achieve a more competitive and integrated EU market for data storage and/or processing services and activities." (EU Regulation, 2018). To meet this goal the Regulation covers the following three issues:

- Improving the mobility of non-personal data across borders in the single market, which is limited today in many Member States by localisation restrictions or legal uncertainty in the market;

- Ensuring that the powers of competent authorities to request and receive access to data for regulatory control purposes, such as for inspection and audit, remain unaffected and

- Making it easier for professional users of data storage or other processing services to switch service providers and to port data, while not creating an excessive burden on service providers or distorting the market.

The Regulation consists of ten articles. The Article 6 which is named "porting of data" prompts Cloud stakeholders to write a self regulatory Code of Conduct (Code) at Union level that covers the guidelines on technical, legal and economical best practices for the switching of cloud providers. As consequence, the article 
6 spurred the creation of the Digital Single Market (DSM) Cloud Switching and Porting Data (SWIPO) Stakeholder Working group which is responsible to develop this Code.

DSM translates the strategy of providing digital opportunities for people and business into policy actions and enhance Europes position in the digital economy (Havu, 2017). DSM provides an environment of fair competition, consumer and data protection, removing geo-blocking and copyright issues based on better access to digital goods and innovative services (Martens, 2013).

The free flow of non-personal data is vital for a competitive data economy and sets the goal that users and companies to be capable to migrate their cloud stored data on their premises or to a different cloud server providers on an easy way with seamless operations and overcome the difficulties of legal uncertainty, lack of trust and the various technical difficulties of service provides (Kouatli, 2016).

The Code covers technical and economical aspects. It constitutes a structured and standardised tool for the stakeholders to communicate and assess the various procedures that should be followed by the Cloud Service Providers (CSPs), the Cloud Service customers (CSCs) and the intermediaries. In the following paragraphs we give a comprehensive relative work (Section 2), we make a description and an analysis on the Code (section 3), we describe the impact of the Code to the CSCs and CSPs (section 4). We cast a critical view on the Code (section 5), and make a review on the models that can estimate the economic impact of the Code in the cloud market (section 6) and discuss our conclusions and future work (section 7).

\section{RELATED WORK}

A Code of Conduct defines how a companys or industrys employees or companies should act on a day-to-day basis (Taylor et al., 2019). It reflects the daily operations, core values and overall culture. As a result, every Code of conduct is unique to the organization or industry it represents and involves ethical principles, company values, accountability, disciplinary actions, and standard of conduct and practises.

A Code of Conduct has been drafted related to the consumer data processing by business and governments (Payne et al., 2015). This Code takes into consideration the desire of consumers for privacy and the businesses for extracted knowledge with data mining methods. It states that a good developed Code, from the one side it maintains some level of privacy and from the other side it can provide better services and improve the economy. At European Union level, Codes related to the Information Community Technology, and specifically data centers and cloud infrastructures has been prepared and drafted. The Code of Conduct for Data Centre Energy Efficiency (FPFIS, 2018) is a voluntary initiative related to the increasing energy consumption in data centres and documents best practises for energy management in order to promote the positive economic and environmental the decrease of power usage of facilities would have. An analysis of the aforementioned Code (Avgerinou et al., 2017) reveals that the average power usage effectiveness of the facilities that follow the Code is constantly improved and confirms that Codes could be effective and of great benefit for European Enterprises and citizens. Additionally, a Code of Conduct for Data Portability and Cloud Service Switching for Infrastructure as a Service (IaaS) Cloud services has been prepared and drafted by a working group of experts.

Why suddenly the need for Codes of Conduct? The answer is pretty simple. Even though the adoption of cloud computing by European enterprises specially is steadily growing, it has not been as big as it would have been expected due to specific barriers faced by Cloud Service Customers (CSCs) such as lack of trust, uncertainty about the applicable law, unclear compliance to existing schemes and legislation, as well as the lack of interoperability among service providers, of transparency in security aspects and of control of the data.

In an attempt to overcome the barriers mentioned above, the ISO/IEC 27018 (de Hert et al., 2016) standard has been published to address the the lack of trust and transparency and to improve the data protection and security challenges of cloud computing. In addition, the European General Data Protection Regulation (GDPR) (Díaz, 2016) gave control to individuals over their personal data and the data portability. on-personal data though is a topic not covered by GDPR, and even more so the non-personal data porting and switching between cloud providers or between on-premise facilities and cloud providers. The Code of Conduct presented in the following section comes, among other things, to cover that topic also.

As it has been very accurately stated in a relevant working paper (Bayrak et al., 2011) Despite the wide consensus that cloud computing is a new and disruptive general purpose technology, there does not seem to be a comparable consensus in denying what exactly cloud computing is, nor a common understanding of how it affects economic fundamentals. Additionally, a study carried out for the European Commission in 2014 
(Deloitte, 2017) provides an overview of how the cloud computing industry has developed in Europe, focusing in the absence of policy measures, and of the most important barriers for its further development. The same study also provides an assessment of the likely impacts (costs and benefits) of policy measures supporting cloud computing to be implemented consistently with the free flow of data initiative recently launched by the Commission.

Deloittes study also confirms the positive impact most guess but cannot prove: a positive economical impact of cloud computing for the EU Estimates regarding the impact of cloud computing adoption to the macro-economic performance of the EU (including impact on GDP) vary. All of them, however, agree in identifying a positive impact. It is estimated that over the next five years, cloud computing could add a cumulative total revenue of EUR 449 billion to the EU28 GDP (including in the public sector). Of these, EUR 57.7 billion and EUR 103.2 billion would be net new GPD generated in the years 2016 and 2020 respectively, representing a share of 0.4 and $0.71 \%$ of total EU GDP respectively5. It is estimated that in 2013, the adoption of cloud computing contributed EUR 27.9 billion to EU GDP, making up $0.2 \%$ of total EU GDP6.

The work presented here deliberates what effect a Code of Conduct for Data Portability and Cloud Service Switching for IaaS Cloud services would have if included in the Certification Scheme for Cloud Computing already being prepared by the EC and based on a few economic models also presented here, the economic impact it might have.

\section{ANALYSIS OF THE CODE OF CONDUCT}

The purpose of the Code of Conduct is to increase CSCs confidence regarding porting and switching between IaaS cloud services or between on- premise facilities and IaaS cloud services, at a low cost and with minimal disruption. Additionally, the Code supports the European Union Free Flow for non personal data Regulation objectives and promotes its stated purpose to achieve a more competitive and integrated EU market for data storage and/ or processing services and activities.

Objectives of the Code (aligned with the aforementioned ECs Regulation) are:

- Reduce the number and range of the data localization restrictions

- Facilitate cross border availability of data for regulatory control purposes

- Improve the conditions under which users can:

1. switch data storage

2. and/ or processing service providers

3. part their data back to their own IT systems

- Enhance trust in the security of cross border data storage and/ or processing.

Overall, the Code intends to support an open and competitive cloud marketplace, which in turn should drive continued adoption and growth of cloud computing, including multi-cloud and hybrid cloud solutions to widen possibilities in the market. Vendor lock-in is not an acceptable business practice. The main characteristics of the Code are described in the following subsections.

\subsection{Purpose of the Code, Target Audience and Responsibility}

The Code is a voluntary instrument, allowing a CISP to evaluate and demonstrate its adherence to the Code requirements for one or more of its Cloud Services by a third party certification or by self-assessment. It intends to provide a set of high level requirements for CISPs, adherence to which will improve a CSCs confidence in achieving an open, transparent and in a meaningful manner successful transfer request of their Infrastructure Artefacts from one cloud service to another or more or between a CISP and a on premise facility.

The target audience of the Code is any party who has an interest in provision of IaaS cloud services (CISPs, CSCs, third party system integrators). The Code does not impose any obligation on the CSC, but recommends that a CSC:

- Be aware of the exit conditions for the discontinuation of a cloud service before entering into a contract with a CISP.

- Develops a cloud service migration plan in anticipation of such an operation. 
- Ensures that the source and destination cloud service(s) can meet their needs with regard to infrastructure portability and cloud service switching.

\subsection{Cloud Services Model for Data Portability}

There is a wide spectrum of CISPs providing a variety of different cloud services, and data portability approaches may differ between cloud services. The Code is not intended to be a one-size-ts-all response to how data portability is conducted for all cloud services or by all CISPs. There is a different Code of Conduct for the Software as a Service (SaaS) cloud services and it is possible there will also be written a Code of Conduct about the Platform as a Service (PaaS). CISPs adopting the Code identify their cloud services which are compliant.

\subsection{Legal Status - Scope/ Personal Data vs non Personal Data}

The Code does not replace a written agreement (CSA) and does not constitute legal advice. It applies to the transfer CSC Infrastructure Artefacts, including both personal and non personal data. Such mixed data sets, where personal and non personal data are inextricably linked, the application of GDPR should not be prejudiced.

\subsection{Scope/ Policy Aspects of Portability}

The transfer of Infrastructure Artefacts between a source and a target has to comply any applicable legal, organizational and policy framework. It is the responsibility of all parties to ensure applicable laws and appropriate software licensing requirements, as relates to them, are followed before, during and after the transfer. This Code does not cover policy related aspects of portability, though a CISP may provide guidance, especially with respect to licensing.

\section{THE ADHERENCE OF THE CLOUD PROVIDERS AND THE IMPACT ON THE CLOUD CUSTOMERS}

Declaration of adherence with the Code obligates a CISP to comply with all the Code Requirements, as explicitly described in Sections 6,7 and 8 of the Code. The Declaration of Adherence confirms that a specific cloud service complies with the Code and a detailed description of compliance measures along with the Declaration shall be conveyed to the CSC, while the Declaration has to be incorporated into the Portability Public Register.

The CISP must promptly reassess its compliance if any change to the cloud service results in a permanent material change to the Declaration the CSC must be noticed accordingly in due time.

The Code denes the requirements and contractual specications which the CSPs should adhere. The adherence on these responsibilities provides to the CSC the confidence that the CSPs can transfer its data in an open, transparent, predictable and meaningful way. The declaration of a CSP that adheres to the

Code has the prerequisite that it complies with all the following Requirements:

\subsection{Requirements}

The Data Portability and Interoperability and IaaS cloud services switching involves sets of requirements:

Procedural requirements, that dene the processes that should be followed and allow CSCs to retrieve their data from CSPs, to upload their data to CSPs, or to port data between two CSPs. These are the initiation of switching and porting from the CSP when it is a porting source. The initiation of switching and porting to the CSP when it is a destination source. The declaration how the porting takes place. The CSP should advise and agree with the CSP about the terms and charges. The activation of a new CSP service when it is the porting destination. The CSP should manage the porting and switching process. The CSP should make the exit process in the source CSP after the porting process is accomplished.

Data portability, that identies the technical measures and the legal obligations that support the porting process. The source CSP take all the reasonable steps to proceed the data transfer with an easy and secure manner. From the other side, the destination CSP should provide support for the inter- operability between the 
CSCs user functions, business functions and the cloud services and support for the CSC application programmatic access to the ported data. The destination CSP should facilitate the porting in an easily and securely way. These data will be in commonly used structures and machine-readable for- mats, but the CSPs are not required to transform the data in different formats. The transfer of data should follow open standards and protocols and as consequence the CSPs should support commonly used formats and packaging. If the data involves code artefacts, the CSPs should describe how they can execute the service dependencies and environment in which they will be executed. Last but not least the CSP shall provide an interface for data retrieval which can be subject for additional costs.

Scope and compatibility requirements that should be explicity defined in the Cloud Service Agreement (CSA) between the CSP and the CSC. These involve the extent of the data and the cloud service interoperability that concern the CSP switching and ensure that there will be no loss of functionality and high security. Derived data such as security records incident history are not a requirement for the CSP to be ported. But they can be ported if it is clearly agreed and described in the CSA.

Planning requirements that are included in the CSA and concerns performance, testing of APIs and pricing mechanisms. The testing of APIs concern the APIs for porting data and the APIs to access and manage the stored data. The CSA should also define the anticipated volume of data, the appropriate duration for the transfer, the transfer rate, the minimum network bandwidth, and the period the ported data will be available to the CSC. The CSA can also include an allocation of security responsibilities such as access control, authentication of users integrity and confidential through the process.

\subsection{Contractual Specifications}

The role of the Code is not to replace the CSA. The CSA denes the terms between the CSPs and CSCs but the contractual rights and the obligations of the Code are not diminished by the CSA. The CSA can be in writing form: in a single document, a set of documents or online terms and conditions.

Transparency should be ensured by the CSPs, which involves that the terms and conditions should be noticed to the CSCs. In addition, the CSPs should describe to the CSCs all the mechanisms that concern the porting of data, that involve the tools that they use, the available support that the CSPs o ers, descriptions of the data porting processes, how the CSPs handles the CSCs data after the exit process, a description of anyone who has access to the CSCs data through the porting process and a description in any kind of conversion, translation and transfer of the data from a third-party service.

A description of the mechanisms related to the porting of the data should be provided from the CSPs to the CSCs, which should at least involve the procedures, terms and conditions, costs and policies concerning the porting, technical, physical and organisational measures to undertake the porting. The data model, data semantics and schema should be explained for functional and no-functional data, the locations where the data going to be stored and the costs that would be charged by the CSP.

In general, the CSPs should made available to the CSCs all the information related to data portability and in case of any change to the conditions and mechanisms the CSPs should inform the CSCs timely and the CSCs would have the right to terminate the agreement.

\section{A CRITICAL VIEW ON THE CODE OF CONDUCT}

The Code will establish new regulations, processes and activities in the Cloud community. Even though it has not been finalized yet and some topics may change, this part of our work is to consider the weak parts of the Code. The cloud stakeholders should be aware of them and act proactively to reduce the risk of their enterprises and the EC should take them into account while forming the new certification scheme for cloud computing.

The first sections of the Code can be quite confusing, since there is not a specic section dedicated to its purpose and another one to the benefits and risks it may have, as it is customary. As a matter of fact, risks are not mentioned in the Code nor the way that could be possible handled, in case one realizes, by a CSC. Additionally, the reader, unless well informed, can be baffled by the Purpose section, the objectives subsection and the intentions paragraph.

A section that is potentially missing is one about GDPR and how the CISP and CSC are affected. Even though the topic of personal and non-personal data is mentioned, no clear guidelines are given and it is not mentioned how GDPR and the ECs Free Flow of non-personal data Regulation t/ are taken into account in the 
Code Requirements or that they simply are. The internal processes of the CISP regarding GDPR are extremely important and it should be mandatory for the CISP to provide at least a minimum amount of information of how its adhering to the Code.

Another issue that is not clearly defined and explained is the Portability Public Registry and the incentive a CISP will have to register the services adhering to the Code there. It is mentioned that a CISP should register the services(s) that adhere to the Code without mentioning the data required, and only at the end of the text there is a referral that the Registry will be created by the Executive Board (Governance Section). No information is provided on what type of data a CISP will be required to provide, what data will be available to the CSC, in which infrastructure the Registry will be operating from, who is going to be responsible for the quality control of the Registry, etc. and of course why it would be on a CISPs benefit to register.

A better description of how a CSC will be able to le a complaint, anonymously, for a CISPs breach of the Code for a service that is registered for adhering the Code has to be included, as well as the specific steps that will be taken to ensure a resolution. Since the Code is no legal document there should be a way, maybe through the initial subscription to the Registry, that if a complaint is filled the CISP will have to perform certain actions, otherwise it will be deleted from the Registry and a notification will be sent and posted.

Additionally, as it is mentioned, the Code will require continuous adaptation and change. How the intervals of review or the extraordinary circumstances requiring one will be defined, who will initiate the process, how the CSCs and CISPs are to be notified, who will be responsible for checking if the Registry is updated after a new version of the Code is released, are a few of the questions that have to be taken into account before the Code becomes a part of a certification scheme.

Overall, the Code will be extremely helpful and will allow for CSCs to be more con dent when it comes to porting and switching but the EC has to fully adopt, approve and promote it in order for it to have an impact.

A collaboration with ISO will be extremely helpful if the Code was to be mentioned in certifications for CISPs. An impact analysis and a needs assessment report have to be performed (and described in the Code how and when) at certain time intervals after the Code is approved in order to secure that the necessary changes and requirements of both the CSCs and CISPs are taken into consideration in the Codes review process. The Code should provide, as an Annex, a list of links or even an actual template of a CSA that will include the adherence of a service to the Code.

Incentives will have to be provided to the CISPs in order to bent their resistance to additional requirements/ conformities they have to adhere to after all they are private companies aiming at pro $t$ and are not interested in creating internal processes that require more resources.

\section{THE ECONOMIC IMPACT OF THE CODE OF CONDUCT}

Plenty of articles and books have been written about cloud computing the last two decades since the IT (systems and processes) industry transformed significantly in order to keep up with the public, but most importantly, private sector adoption of cloud computing. The recent financial crisis and recession has also contributed to the accelerating adoption of cloud computing as companies have been forced to find cost-effective IT solutions, which was supported by an IDC (2009) survey of 696 IT executives and CIOs where $51 \%$ of respondents reported that the key driver behind adopting or considering cloud computing is the need to cut costs, while another $29 \%$ report that budget issues force them to find cheaper IT solutions (Gartner, 2016). The literature on topic though, economics of IaaS cloud services, is pretty poor and limited and is presented briefly in the following paragraphs. The fact remains though that, in terms of business creation, approximately 303.000 new businesses, in particular SMEs, could be created between 2015 and 2020 through the development and deployment of cloud computing (Marston et al., 2011) .Most articles published on the topic deal with the pricing of the services and how Nashs equilibrium can be used in order for an equilibrium in the sector to be reached - no studies have been published though examining in depth based on economic models the impact of this sector to economy, so only theories can be presented.

According to Etro (Etro, 2015), through cloud computing, firms will be able to rent computing power (both hardware and software in their latest versions) and storage from a service provider and that will have a profound impact on the cost structure of all the industries using hardware and software, and therefore it will have crucial consequences on:

- Business creation and macroeconomic performance; 
- Job creation in all industries and job reallocation in the ICT sector;

- Public finance accounts, through the direct impact on the public sector spending and the indirect one on the tax revenues.

Additionally, Etro in another article (Etro, 2009) is using a DSGE model to perform his calculations and show a significant medium term impact of the diffusion of cloud computing on entry of SMEs, employment and growth. Starting from conservative assumptions on the cost reduction process, the analysis of the calculations based on his model, shows that the diffusion of cloud computing will provide (in a medium term range of ve years) a positive impulse to the annual growth rate, contributing to create about a million new jobs through the development of a few hundred thousand new SMEs in the countries he was investigating. What is highlighted though, is that services maintain market power over providers even when services are highly competitive, and that one should not expect the cloud marketplace to support a large number of providers.

In the The Economics of Cloud Computing (Bayrak et al., 2011) article, a different economic perspective is presented, based on the argument that the technological categorizations used in cloud computing (Software, Platform and Infrastructure as a Service) do not correlate well to the economic impacts of cloud technologies, and the proposal made is that economists should perceive it as wholesale and retail cloud applications. Wholesale cloud applications are primarily aimed at businesses and facilitate large-scale data integration projects, rapid, low-fixed cost entry and expansion of new start-ups, as well as the outsourcing of many noncore aspects of a given firms activities. Retail cloud applications, on the other hand, are primarily aimed at consumers and the fact that they move applications and content off personal computers to various types of cloud platforms and make both consumers produced and purchased content available in increasingly device and location agnostic ways (Bayrak et al., 2011).

Even though cloud computing is understandably by now extremely important, several factors limit the adoption of cloud computing services by professional users (Deloitte, 2017) such as from professional users' prospective data access and control, data portability, and interoperability. From the providers' perspective, these are the local support, procurement rules and for both of them the data localization requirements and information security.

How will the Code of Conduct affect the cloud computing sector, and most importantly, what is its possible economic footprint? Well, according to Gartner (Gartner, 2016), globally, through 2015, more than $90 \%$ of private cloud computing deployments will be for infrastructure as a service and as it is depicted in a gure at Deloittes study (Etro, 2009) the market for private cloud is estimated at EUR 5.9 billion in 2015, and by 2020, the market value of private cloud would be worth EUR 12.06 billion across EU Member States (up to EUR 14.6 billion in the optimistic scenario and EUR 10.3 billion in the pessimistic one). Removing barriers that have been recorded for both consumers and providers of cloud computing services will only benefit this economic sector and can only have positive economic impact.

From the research performed for the purpose of this paper, we suggest that the economic theory that can be used and have usable outcomes, is to perceive and deal with it as wholesale and retail cloud applications, since both consumers and providers will be a affected by the implementation of the Code. Wholesale cloud applications are primarily aimed at businesses: how they will be able to facilitate large-scale data integration projects, rapid low-fixed cost entry and expansion of new start-ups, along with the outsourcing of many non-core aspects of a given firm's activities. Retail cloud applications are primarily aimed at consumers, who are or will move applications and content off on site computers to various types of cloud platforms and make both consumer produced and purchased content available in increasingly device and location agnostic ways (Etro, 2015) .The Code will come and set ground rules on how both of these categories will do business with an aim to remove as many obstacles as possible - both of them will be affected, so a wholesale - retail economic theory has to be applied.

\section{CONCLUSION AND FUTURE WORK}

The Code of Conduct aims to become the basic text that CSCs will be required to have knowledge of in order to be clear of what their contract or CSA includes regarding data portability and switching among CISPs. Additionally, the Code fills the existing gap of requirements that the CISPs should adhere to in order to prove in a very simple and straightforward way that they are in a position to achieve an open, transparent and successful transfer request of Infrastructure Artefacts to another CISP. 
Even though the economic impact of cloud computing will continue to be positive, which is supported by almost all studies and articles, there is no clear picture on the specific sector of IaaS. According to the research performed for this paper, the best option is likely to follow the theory of wholesale - retail to develop an economic model that could be initially used to forecast the economic footprint of a Code for Data Portability and Cloud Service Switching that can be later be followed by a game theory application.

Concluding, let us borrow the conclusion of a very interesting article (Marston et al., 2011) \that states the famous physicist Niels Bohr once said, Prediction is very difficult, especially about the future. The fluid and uncertain environment that surrounds cloud computing be it in terms of the technology, its adoption, the industry structure or the regulatory regime that will eventually need to be in place makes such an exercise that much more difficult. Hopefully, with our work, the bases for new research initiatives regarding economic models for cloud computing and the impact the implementation of new regulations such as a Code will have has been established and new discussions and debates will open up for this specific topic.

\section{REFERENCES}

Avgerinou, M., Bertoldi, P., Castellazzi, L., 2017. Trends in Data Centre Energy Consumption under the European Code of Conduct for Data Centre Energy Efficiency. Energies 10, 1470. https://doi.org/10.3390/en10101470

Bayrak, E., Conley, J., Wilkie, S., 2011. The Economics of Cloud Computing (Vanderbilt University Department of Economics Working Paper No. 1118). Vanderbilt University Department of Economics.

De Hert, P., Papakonstantinou, V., Kamara, I., 2016. The cloud computing standard ISO/IEC 27018 through the lens of the EU legislation on data protection. Computer Law \& Security Review 32, 16-30. https://doi.org/10.1016/j.clsr.2015.12.005

Deloitte, 2017. Measuring the economic impact of cloud computing in Europe [WWW Document]. Digital Single Market - European Commission. URL https://ec.europa.eu/digital-single-market/en/news/measuring-economic-impact-cloudcomputing-europe (accessed 6.7.19).

Díaz, E.D., 2016. The new European Union General Regulation on Data Protection and the legal consequences for institutions. Church, Communication and Culture 1, 206-239. https://doi.org/10.1080/23753234.2016.1240912

Etro, F. 2009. The Economic Impact of Cloud Computing on Business Creation, Em- ployment and Output in Europe. An application of the Endogenous Market Struc- tures Approach to a GPT innovation. In Review of Business and Economic Litera- ture, KU Leuven, Faculty of Economics and Business, pages 179-208.

Etro, F. 2015. The Economicsof Cloud Computing. InI.ManagementAssociation (Ed.), Cloud Technology: Concepts, Methodologies, Tools, and Applications. Hershey, PA: IGI Global. https://doi.org/10.4018/978-1-4666-6539-2.ch101

Forecast Overview: Public Cloud Services, Worldwide, 2011-2016, 4Q12 Update [WWW Document], n.d. . Gartner. URL https://www.gartner.com/en/documents/2332215/forecast-overview-public-cloud-services-worldwide-2011-2 (accessed 6.7.19).

FPFIS, 2018. 2018 Best Practice Guidelines for the EU Code of Conduct on Data Centre Energy Efficiency: Version 9.1.0 [WWW Document]. EU Science Hub - European Commission. URL https://ec.europa.eu/jrc/en/publication/2018-bestpractice-guidelines-eu-code-conduct-data-centre-energy-efficiency-version-910 (accessed 6.4.19).

Havu, K., 2017. THE EU DIGITAL SINGLE MARKET FROM A CONSUMER STANDPOINT: HOW DO PROMISES MEET MEANS? Contemporary Readings in Law and Social Justice 9, 146-183.

Implementing Codes of Conduct | How Businesses Manage Social Performance in Global Supply Chains | Taylor \& Francis Group [WWW Document], n.d. URL https://www.taylorfrancis.com/books/9781351280723 (accessed 5.24.19).

Kouatli, I., 2016. Managing Cloud Computing Environment: Gaining Customer Trust with Security and Ethical Management. Procedia Computer Science, Promoting Business Analytics and Quantitative Management of Technology: 4th International Conference on Information Technology and Quantitative Management (ITQM 2016) 91, 412-421. https://doi.org/10.1016/j.procs.2016.07.110

Marston, S., Li, Z., Bandyopadhyay, S., Zhang, J., Ghalsasi, A., 2011. Cloud computing - The business perspective. DECISION SUPPORT SYSTEMS 51, 176-189.

Martens, B., 2013. What Does Economic Research Tell Us About Cross-Border E-Commerce in the EU Digital Single Market? (SSRN Scholarly Paper No. ID 2265305). Social Science Research Network, Rochester, NY.

Payne, D.M., Landry, B.J.L., Dean, M.D., 2015. Data Mining and Privacy: An Initial Attempt at a Comprehensive Code of Conduct for Online Business. CAIS 37, 34. https://doi.org/10.17705/1 cais.03734

Regulation (EU) 2018/1807 of the European Parliament and of the Council of 14 November 2018 on a framework for the free flow of non-personal data in the European Union (Text with EEA relevance.), 2018., OJ L. 